Classification

Physics Abstracts

$66.30 \mathrm{~J}-07.62$

\title{
Diffusion du zinc dans GaAlSb et application à la photodétection infrarouge
}

\author{
A. Joullié, F. De Anda, P. Salsac \\ Equipe de Microoptoélectronique de Montpellier $\left(\mathrm{EM}^{2}\right)$, \\ Université des Sciences et Techniques du Languedoc, 34060 Montpellier Cedex, France \\ et M. Mebarki \\ Institut des Sciences Exactes, Université d’Oran, Algérie
}

(Reçu le 14 juin 1983, révisé le 9 novembre, accepté le 12 novembre 1983)

\begin{abstract}
Résumé. - La diffusion du zinc a été effectuée dans les alliages $\mathrm{Ga}_{1-x} \mathrm{Al}_{x} \mathrm{Sb}$ de type $\mathrm{n}$, à $x \leqslant 0,20$, obtenus par épitaxie en phase liquide sur substrat $\mathrm{GaSb}(111)$. Les températures de diffusion ont été fixées à $510^{\circ} \mathrm{C}, 550^{\circ} \mathrm{C}$ et $600^{\circ} \mathrm{C}$. La diffusion a été réalisée en tube fermé à partir de la phase vapeur. Les profils de diffusion ont été déterminés avec une microsonde ionique. Le coefficient de diffusion effectif a été calculé. Il s'écrit $D_{\text {eff }}=D_{0} \exp \left(-E_{0} / k T\right)$ avec pour $x=0,17, D_{0}=8 \mathrm{~cm}^{2} \mathrm{~s}^{-1}$ et $E_{0}=1,92 \mathrm{eV}$. La solubilité limite ainsi que la vitesse de diffusion du zinc augmentent avec la teneur en aluminium de l'alliage. Des photodiodes $\mathrm{p}^{+} \mathrm{n}$ à géométrie MESA $(\varnothing=300 \mu \mathrm{m})$ ont été préparées. Les jonctions diffusées sont abruptes avec des courants inverses (autour de $10 \mu \mathrm{A}$ ) attribués à des courants de surface. L'étude de la réponse spectrale des photodiodes a été effectuée en fonction de la composition de l'alliage et de la profondeur de jonction. Les meilleurs résultats ont été obtenus avec des jonctions peu diffusées $(0,4 \mu \mathrm{m})$. Ils montrent que les structures diffusées $\mathrm{p}^{+} \mathrm{n}$ à $\mathrm{Ga}_{0,85} \mathrm{Al}_{0,15} \mathrm{Sb}$ et $\mathrm{Ga}_{0,96} \mathrm{Al}_{0,04} \mathrm{Sb}$ sont adaptées à la photodétection à $1,3 \mu \mathrm{m}$ et $1,55 \mu \mathrm{m}$.

Abstract. - Zinc diffusion was performed into $\mathrm{n}$ type $\mathrm{Ga}_{1-x} \mathrm{Al}_{x} \mathrm{Sb}$ alloys $(x \leqslant 0.20)$ grown by liquid phase epitaxy onto (111) GaSb substrates. The diffusion temperatures were $510^{\circ} \mathrm{C}, 550^{\circ} \mathrm{C}$ and $600^{\circ} \mathrm{C}$. The diffusion was carried out in a closed system from zinc vapour phase. The diffusion profiles were determined with the help of a ionic microprobe. The effective diffusion coefficient was calculated. It is expressed as $D_{\text {eff }}=D_{0} \exp \left(-E_{0} / k T\right)$ where $D_{0}=8 \mathrm{~cm}^{2} \mathrm{~s}^{-1}$ and $E_{0}=1.92 \mathrm{eV}$ for $x=0.17$. The zinc solubility limit and the zinc diffusion rate increased with the aluminium concentration of the alloy. MESA p ${ }^{+} \mathrm{n}$ photodiodes were prepared $(\varnothing=300 \mu \mathrm{m})$. Diffused junctions are abrupt, with reverse currents (about $10 \mu \mathrm{A}$ ) due to surface leakage. The spectral response was studied as a function of the alloy composition and junction depth. The best results were obtained with shallow junctions $(0,4 \mu \mathrm{m})$. They show that $\mathrm{Ga}_{0.85} \mathrm{Al}_{0.15} \mathrm{Sb}$ and $\mathrm{Ga}_{0.96} \mathrm{Al}_{0.04} \mathrm{Sb}$ diffused $\mathrm{p}^{+} \mathrm{n}$ structures are adapted to $1.3 \mu \mathrm{m}$ and $1.55 \mu \mathrm{m}$ photodetection.
\end{abstract}

\section{Introduction.}

Le développement des fibres optiques avec des caractéristiques d'atténuation et de dispersion fortement améliorées dans le domaine des longueurs d'ondes centré autour de $1,3 \mu \mathrm{m}$ et $1,55 \mu \mathrm{m}[1,2]$ a stimulé la recherche de nouveaux matériaux pour composants d'extrémité. Parmi ceux-ci les systèmes GaAlSb/GaSb et $\mathrm{GaAlAsSb} / \mathrm{GaSb}$ paraissent très prometteurs, surtout pour les photodétecteurs [ 3 à 8 ]. Ces derniers ont été le plus souvent réalisés à partir de jonctions du type $\mathrm{p}^{+} \mathrm{n}$ où la zone $\mathrm{p}^{+}$est obtenue par épitaxie en phase liquide d'une couche dopée au germanium $[3,7]$, par implantation ionique de béryllium $[5,8]$ ou par diffusion de zinc [9].
Divers résultats ont été publiés concernant la diffusion du zinc dans GaSb [9 à 12] et GaAlAsSb [9, 13]. Fort peu concernent le ternaire GaAlSb [14].

Dans cet article, nous décrivons nos essais de diffusion du zinc, à partir de la phase vapeur, dans les composés ternaires $\mathrm{Ga}_{1-x} \mathrm{Al}_{x} \mathrm{Sb}$ de type $\mathrm{n}$ élaborés par épitaxie en phase liquide, ainsi que la préparation, pour la première fois, de photodiodes MESA diffusées $\mathrm{p}^{+} \mathrm{n}$ à GaAlSb.

Les propriétés photoélectriques de telles structures sont indiquées ainsi que le rôle de la composition de l'alliage et de la profondeur de jonction sur la réponse spectrale. 


\section{Diffusion du zinc dans GaAlSb.}

2.1 TeChNique OPÉRATOIRE. - Pour réaliser la diffusion du zinc dans GaAlSb, nous avons utilisé la méthode de diffusion à partir de la phase vapeur en enceinte fermée. Cette méthode est couramment utilisée pour diffuser du zinc dans les semi-conducteurs III-V $[15,16]$. Sa technologie est simple et si elle ne permet pas de traiter un grand nombre de plaquettes, comme cela est possible avec les techniques de diffusion en tube ouvert, elle permet de s'affranchir des problèmes de contrôle des pressions de vapeur partielles, tout en obtenant une bonne reproductibilité des résultats.

Le matériau à diffuser est constitué par des couches de $\mathrm{GaSb}$ et de $\mathrm{Ga}_{1-x} \mathrm{Al}_{x} \mathrm{Sb}(x \leqslant 0,20)$ obtenues par épitaxie en phase liquide. Celle-ci a été réalisée sur substrat $\mathrm{GaSb}$ orienté (111) B fortement dopé au tellure $\left(3 \times 10^{18} \mathrm{~cm}^{-3}\right)$ avec - ou pas - la présence d'une couche intermédiaire de $\mathrm{GaSb}$ également très dopée avec du tellure (couche tampon). Les couches épitaxiées sont plus faiblement dopées $(8-10 \times$ $10^{16} \mathrm{~cm}^{-3}$ ). La densité de dislocations de ces couches est de 1 à $5 \times 10^{5} \mathrm{~cm}^{-2}$, indépendante de la teneur en aluminium. Après épitaxie, les surfaces de dépôts sont nettoyées au brome méthanol (à $1 \%$ ) pendant une dizaine de secondes.

L'échantillon, une fois rincé à l'alcool puis séché, est placé dans un tube en quartz comportant à son extrémité un logement qui contient une pastille de zinc. Le poids de ce zinc est très supérieur à celui nécessaire pour atteindre la pression de saturation à la température de diffusion. Le tube est ensuite vidé jusqu'à une pression de $5 \times 10^{-6}$ torr. Le volume utile est de $10 \mathrm{~cm}^{3}$.

Le tube est alors rapidement placé dans un four à résistance régulé à $\pm 0,5^{\circ} \mathrm{C}$. $\mathrm{La}$ température de l'échantillon (température de diffusion) est supérieure de $10^{\circ} \mathrm{C}$ à celle du zinc (zone froide), ceci afin d'éviter tout dépôt de zinc sur l'échantillon.

Les diffusions ont été réalisées à trois températures différentes : 510,550 et $600^{\circ} \mathrm{C}$, pendant des durées variant de $10 \mathrm{~min}$ à $4 \mathrm{~h}$.

2.2 RÉSULTATS EXPÉRIMENTAUX. - La surface après diffusion reste brillante mais son type de conductivité est devenu p. Le front de diffusion est plat. Il est révélé par attaque chimique $-20 \mathrm{H}_{2} \mathrm{O} / 1$ $\mathrm{KOH} / 1 \mathrm{~K}_{3}(\mathrm{FeCN})_{6}$ - de clivages ou, dans le cas des diffusions peu profondes, de biseaux. Sa position vis-à-vis de la surface permet de définir la profondeur de diffusion que nous avons assimilée à la profondeur de jonction $x_{\mathrm{j}}$. Un exemple d'homojonction à GaAlSb, ainsi obtenue par diffusion de zinc, est présenté figure 1.

La variation de la profondeur de jonction $x_{\mathrm{j}}$ avec la durée $t$ de la diffusion est présentée figure 2 dans le cas des alliages $\mathrm{Ga}_{0,83} \mathrm{Al}_{0,17} \mathrm{Sb}$, pour différentes températures de diffusion.

La figure 3 indique la variation de $x_{\mathrm{j}}$ avec $t$ pour

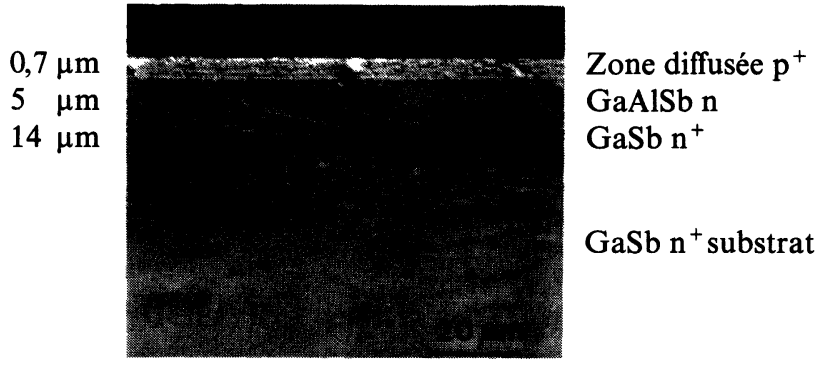

Fig. 1. - Structure avec couche tampon et zone diffusée $\mathrm{p}^{+}$. Attaque chimique $20 \mathrm{H}_{2} \mathrm{O}-1 \mathrm{KOH}-1 \mathrm{~K}_{3}(\mathrm{FeCN})_{6}$ durée $2 \mathrm{~s}$.

[Diffused structure with a buffer layer (chemical revelation $\left.20 \mathrm{H}_{2} \mathrm{O}-1 \mathrm{KOH}-1 \mathrm{~K}_{3}(\mathrm{FeCN})_{6} ; 2 \mathrm{~s}\right)$.]

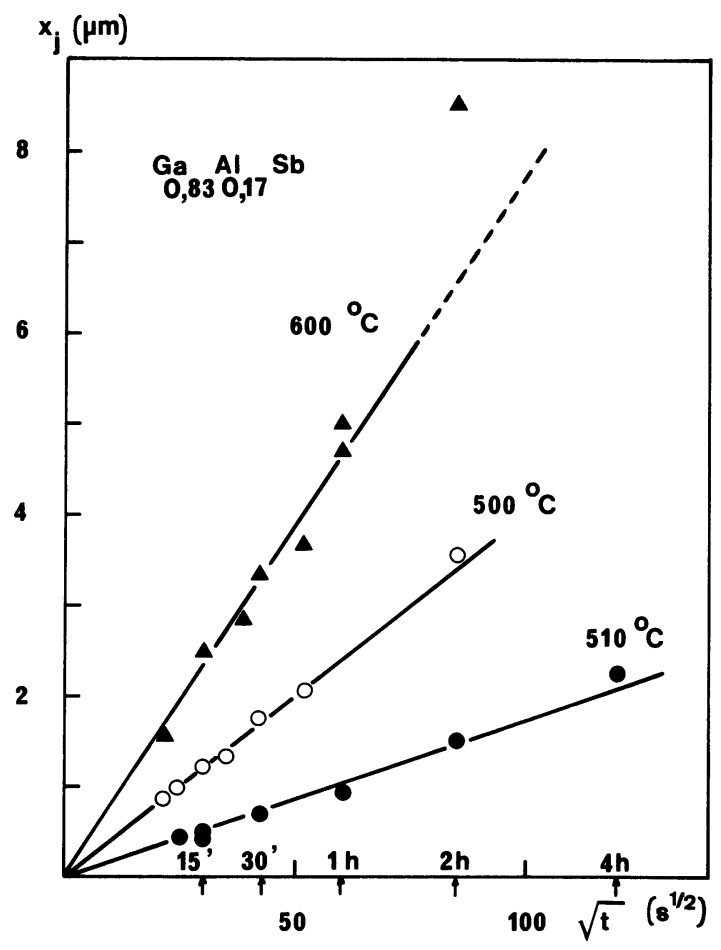

Fig. 2. - Variation de la profondeur de jonction $x_{\mathrm{j}}$ avec la durée $t$ de la diffusion dans $\mathrm{Ga}_{0,83} \mathrm{Al}_{0,17} \mathrm{Sb}$.

[Variation of the junction depth $x_{\mathrm{j}}$ with the diffusion time $t$ for $\mathrm{Ga}_{0.83} \mathrm{Al}_{0.17} \mathrm{Sb}$.]

des alliages de compositions différentes dans le cas des diffusions à $510^{\circ} \mathrm{C}$.

Sur la figure 4, nous comparons nos résultats à ceux publiés concernant la diffusion du zinc dans $\mathrm{GaSb}$ et $\mathrm{Ga}_{0,70} \mathrm{Al}_{0,30} \mathrm{As}_{0,03} \mathrm{Sb}_{0,97}$ à partir de $\mathrm{Zn}-\mathrm{Sb}$ et $\mathrm{Zn}$-As [9], ou de $\mathrm{Zn}_{0,07} \mathrm{Ga}_{0,93}$ [13].

Les profils de diffusion du zinc ont été déterminés avec un analyseur ionique (Cameca). La zone décapée par bombardement d'ions $\mathrm{O}_{2}^{+}$est un carré de $900 \mu \mathrm{m}$ de côté. Les ions $\mathrm{Zn}^{+} 64$ émis normalement à la surface (sur un diamètre de $400 \mu \mathrm{m}$ ) sont recueillis, une partie de ce faisceau (sur un diamètre de $100 \mu \mathrm{m}$ ) est analysée. 


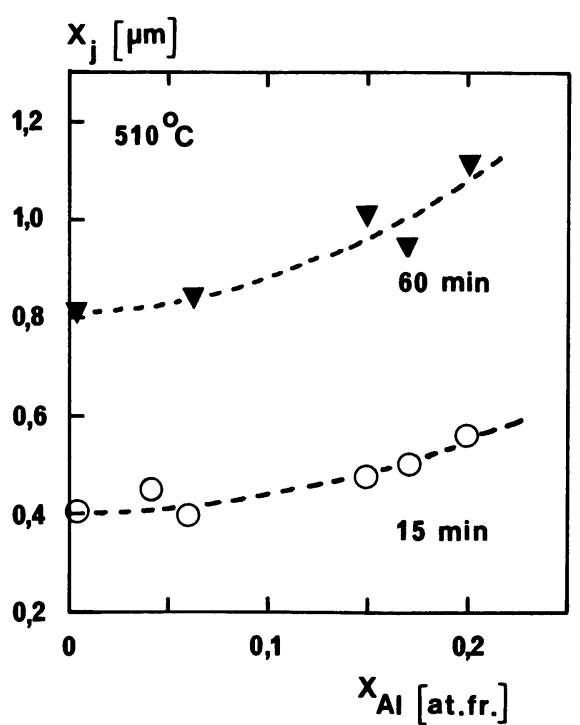

Fig. 3. - Variation de la profondeur de jonction $x_{\mathrm{j}}$ avec la concentration en aluminium pour des diffusions à $510^{\circ} \mathrm{C}$.

[Variation of the junction depth $x_{j}$ with the aluminium concentration for diffusions performed at $510^{\circ} \mathrm{C}$.]

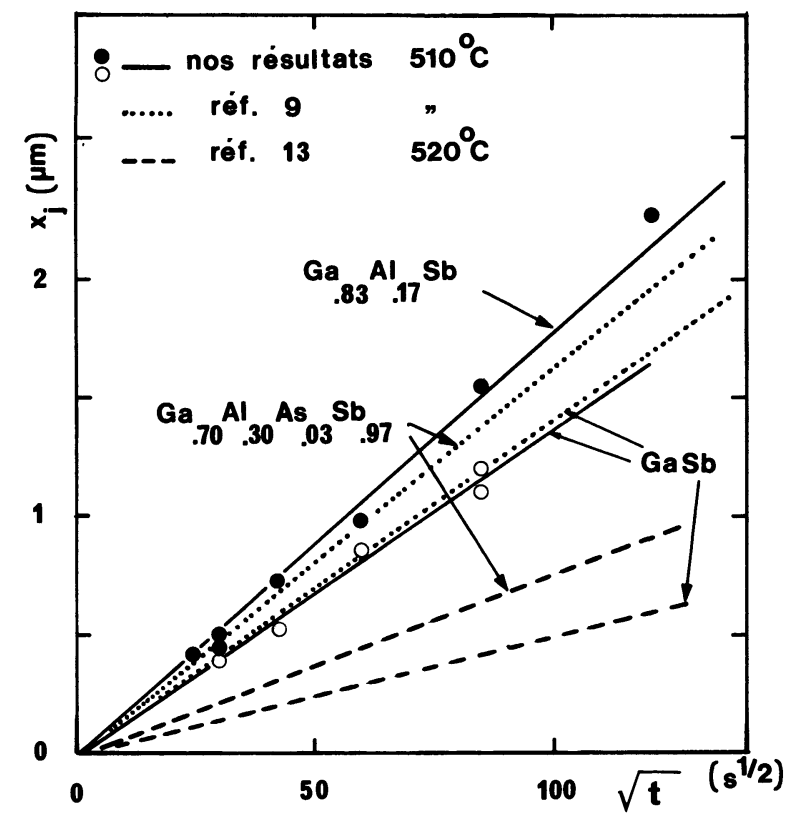

Fig. 4. - Variation de la profondeur de jonction $x_{\mathbf{j}}$ avec $t$ pour différents alliages.

[Variation of the junction depth $x_{\mathbf{j}}$ with $t$ for different alloys.]

La vitesse de décapage a été déterminée en mesurant, au Talysurf, la profondeur décapée au bout d'une heure dans un échantillon témoin. La vitesse de décapage est de $10,75 \AA / \mathrm{s}$. Les courbes présentées figure 5 donnent les profils de diffusion du zinc pour des diffusions effectuées à $510^{\circ} \mathrm{C}$, de $15 \mathrm{~min}, 1 \mathrm{~h}$ et $2 \mathrm{~h}$. Les courbes présentées figures 6 et 7 montrent les profils de diffusion obtenus à des températures différentes avec les alliages $\mathrm{Ga}_{0,83} \mathrm{Al}_{0,17} \mathrm{Sb}$.

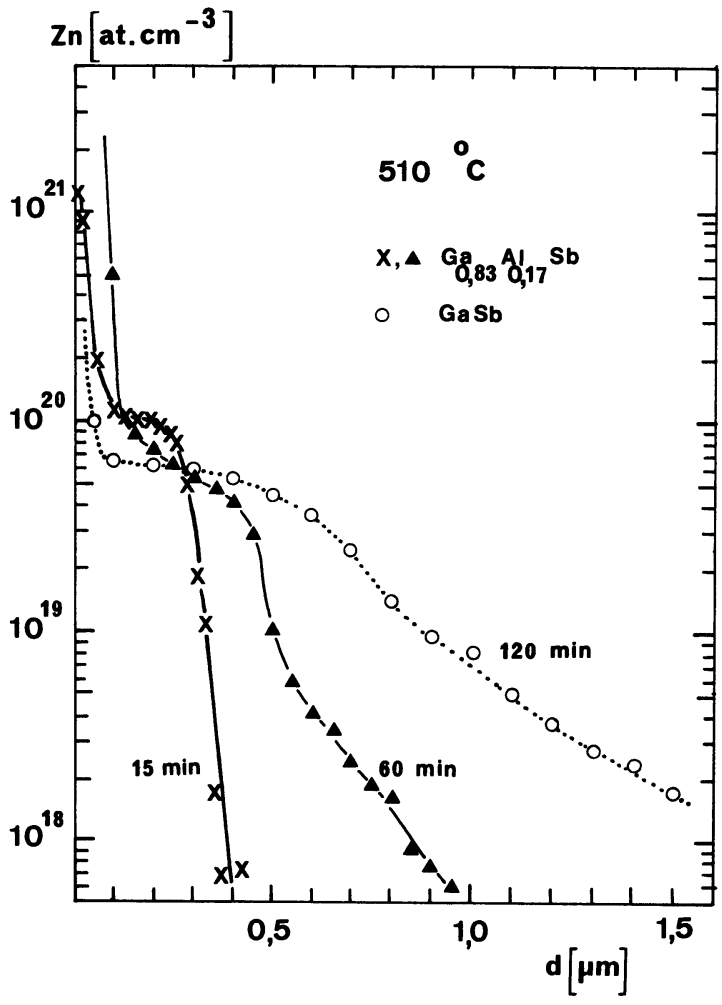

Fig. 5. - Profils de concentration en zinc pour des diffusions à $510^{\circ} \mathrm{C}$.

[Zinc concentration profiles for diffusions performed at $510^{\circ} \mathrm{C}$.]

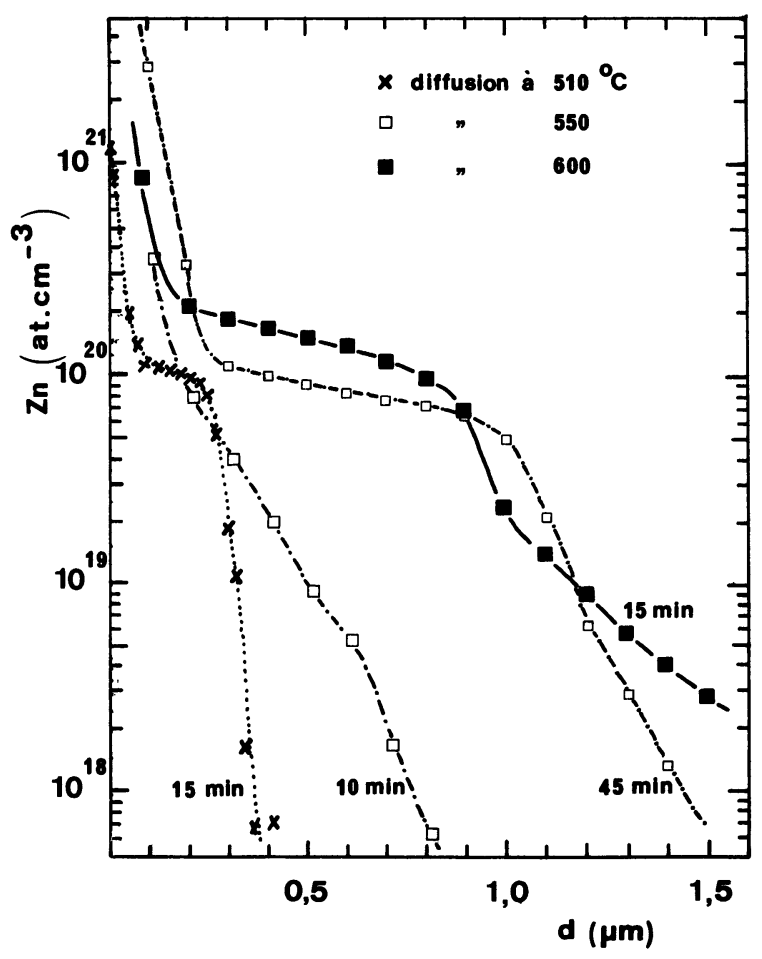

Fig. 6. - Profils de concentration en zinc pour des diffusions dans $\mathrm{Ga}_{0,83} \mathrm{Al}_{0,17} \mathrm{Sb}$.

[Zinc concentration profiles for diffusions into $\mathrm{Ga}_{0.83} \mathrm{Al}_{0.17} \mathrm{Sb}$.] 


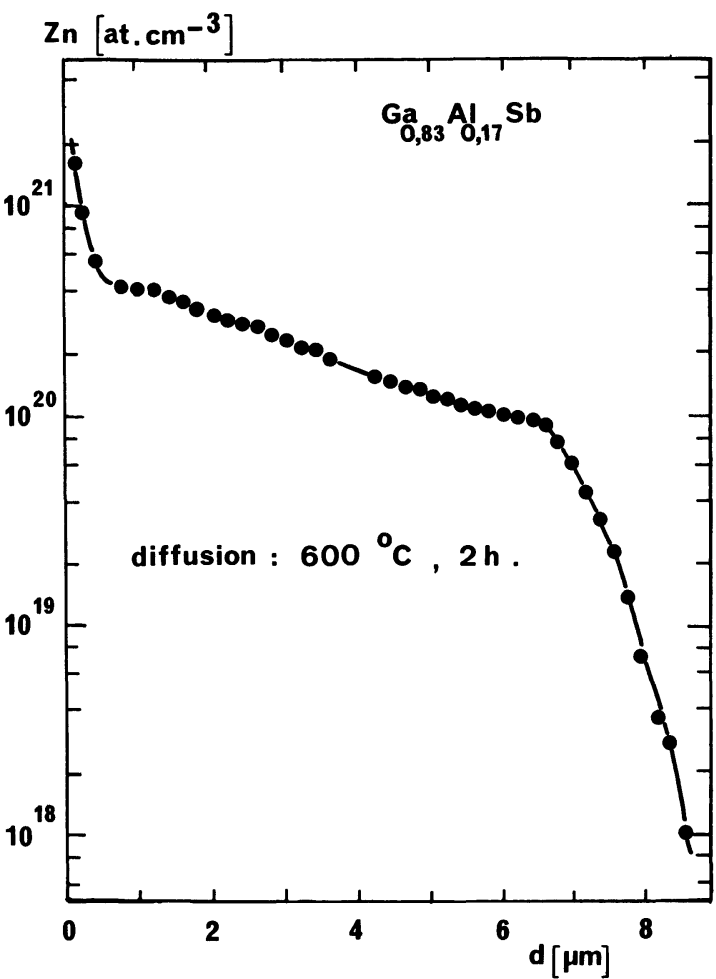

Fig. 7. - Profil de concentration en zinc après une diffusion de 2 heures à $600{ }^{\circ} \mathrm{C}$ dans $\mathrm{Ga}_{0,83} \mathrm{Al}_{0,17} \mathrm{Sb}$.

[Zinc concentration profile for a 2 hours $600^{\circ} \mathrm{C}$ diffusion into $\mathrm{Ga}_{0.83} \mathrm{Al}_{0.17} \mathrm{Sb}$.]

Ces profils font apparaître trois zones distinctes :

- Une zone de quelques fractions de micron où il y a une brutale augmentation de la concentration en zinc. Ceci est probablement le fait d'une exaltation du signal due à la pollution de surface (en particulier, présence d'oxygène). Une attaque chimique par $\mathrm{HCl}$ (réduction de l'oxygène) et une décontamination de la surface (vide prolongé) ont pour effet de réduire l'importance de cette « queue » de surface à $\simeq 0,15 \mu \mathrm{m}$.

- Une zone de décroissance lente et régulière de la concentration en zinc. La longueur de cette zone est directement liée à la durée de la diffusion.

- Une zone où la concentration chute brutalement et qui se traduit par l'existence d'un front de diffusion.

La mesure quantitative de la concentration en zinc a été effectuée à la microsonde électronique de Castaing sur deux couches témoins à grande profondeur de jonction, après décapage au brome-méthanol de la zone superficielle $(1 \mu \mathrm{m})$. Il est alors possible de se fixer une échelle en ordonnées exprimée en at $/ \mathrm{cm}^{3}$. Cette échelle montre que la limite de la détection du zinc varie autour de $5 \times 10^{17}$ at. $\mathrm{cm}^{-3}$.

Le dopage $\mathrm{n}$ des couches étant de l'ordre de $10^{17} \mathrm{~cm}^{-3}$, la profondeur de jonction peut être évaluée à partir des profils de diffusion par extrapolation des couches de concentration en zinc, jusqu'à la valeur $10^{17}$ at $\mathrm{cm}^{-3}$. Le tableau I montre que les profondeurs ainsi déterminées sont comparables à celles mesurées optiquement à partir du front de diffusion.
Tableau I. - Profondeurs de diffusion $x_{\mathrm{j}} d u$ zinc dans $\mathrm{Ga}_{0,83} \mathrm{Al}_{0,17} \mathrm{Sb}$ déterminées au microscope optique après révélation chimique de la jonction $\mathrm{p}^{+} \mathrm{n}$, comparées à celles obtenues par extrapolation des profils de concentration en zinc jusqu'à la valeur $10^{17}$ at. $\mathrm{cm}^{-3}$. (Les valeurs fournies par cette seconde méthode sont très approchées).

[Junctions depths of zinc in $\mathrm{Ga}_{0.83} \mathrm{Al}_{0.17} \mathrm{Sb}$ determined from the optical microscope after chemical revelation of the $\mathrm{p}^{+} \mathrm{n}$ junction, compared with values obtained by extrapolation of the zinc concentration profiles until $10^{17}$ at. $\mathrm{cm}^{-3}$ (those last values are approximative).]

\begin{tabular}{|c|c|c|c|}
\hline \multirow{2}{*}{$\begin{array}{c}T \text { diffusion } \\
\left({ }^{\circ} \mathrm{C}\right)\end{array}$} & \multirow{2}{*}{$\begin{array}{l}\text { Durée de la } \\
\text { diffusion } \\
\text { (s) }\end{array}$} & \multicolumn{2}{|c|}{$\begin{array}{l}\text { Profondeur de diffusion } \\
\qquad(\mu \mathrm{m})\end{array}$} \\
\hline & & $\begin{array}{l}\text { Analyse } \\
\text { chimique }\end{array}$ & $\begin{array}{l}\text { Analyse } \\
\text { sonde } \\
\text { ionique }\end{array}$ \\
\hline 600 & 450 & 1,55 & 1,7 \\
\hline - & 900 & 2,5 & 2,6 \\
\hline- & 1500 & 2,85 & \\
\hline- & 1800 & 3,3 & \\
\hline - & 2700 & 3,7 & \\
\hline - & 3600 & 4,7 & \\
\hline - & 3600 & 5 & \\
\hline- & 7200 & 8,5 & 9,5 \\
\hline 550 & 450 & 0,85 & 0,9 \\
\hline- & 600 & 1 & 0,95 \\
\hline - & 900 & 1,2 & \\
\hline- & 1200 & 1,3 & \\
\hline - & 1800 & 1,7 & \\
\hline- & 2700 & 2,1 & 1,6 \\
\hline 510 & 600 & 0,45 & \\
\hline - & 900 & 0,45 & 0,4 \\
\hline - & 900 & 0,5 & \\
\hline - & 1800 & 0,7 & \\
\hline- & 3600 & 0,95 & 1,2 \\
\hline- & 7200 & 1,5 & \\
\hline- & 16400 & 2,3 & \\
\hline
\end{tabular}

2.3 CARACTÉRISATION ÉLECTRIQUe DES COUCHES DIFFUSÉES. - Les couches diffusées ont une épaisseur définie comme étant la profondeur de jonction $x_{\mathrm{j}}$. Elles sont fortement conductrices et les zones non diffusées peuvent être considérées comme isolantes vis-à-vis des zones diffusées.

Des mesures de résistivité et d'effet Hall ont été effectuées sur des couches diffusées à grande profondeur de jonction $(2$ à $3 \mu \mathrm{m})$. Les contacts ohmiques ont été réalisés sur la surface par évaporation d'or, sans recuit, selon la méthode de Van der Paw.

Les caractéristiques électriques des couches diffusées se sont avérées peu liées à la température de diffusion. La concentration en porteurs est de l'ordre de $10^{20} \mathrm{~cm}^{3}$, avec une faible mobilité de Hall $\left(50 \mathrm{~cm}^{2} \mathrm{~V}^{-1} \mathrm{~s}^{-1}\right)$. La conductivité est d'environ $10^{3} \Omega^{-1} \mathrm{~cm}^{-1}$. Elle est très supérieure à celle de la 
zone $\mathbf{n}$ du dépôt non diffusé de GaAsSb qui est de l'ordre de $50 \Omega^{-1} \mathrm{~cm}^{-1}$, ce qui valide les mesures effectuées.

2.4 Discussion. - La profondeur de jonction varie linéairement avec $V \bar{t}$ ainsi que le prévoient les théories classiques de la diffusion (source constante, milieu semi-infini), dans GaSb comme dans GaAlSb.

Les coefficients de diffusion effectifs, définis par $D_{\text {eff }}=x_{\mathrm{j}}^{2} / t$, sont, pour $\mathrm{Ga}_{0,87} \mathrm{Al}_{0,17} \dot{\mathrm{Sb}}$ et $\mathrm{GaSb}$ à $510^{\circ} \mathrm{C}$, respectivement égaux à $3,2 \times 10^{-12} \mathrm{~cm}^{2} \mathrm{~s}^{-1}$ et $1,9 \times 10^{-12} \mathrm{~cm}^{2} \mathrm{~s}^{-1}$. La variation de $D_{\text {eff }}$ avec la température de diffusion est exprimée figure 8 . Elle est de la forme :

$$
D_{\text {eff }}=D_{0} \exp \left(-E_{0} / k T\right)
$$

avec pour $\mathrm{Ga}_{0,83} \mathrm{Al}_{0,17} \mathrm{Sb}: D_{0}=8 \mathrm{~cm}^{2} \mathrm{~s}^{-1}$ et $E_{0}=1,92 \mathrm{eV}$.

Ces résultats sont comparables à ceux obtenus par T. Kagawa et G. Motosugi [9] concernant la diffusion de zinc dans $\mathrm{GaSb}$ et $\mathrm{Ga}_{0,70} \mathrm{Al}_{0,30} \mathrm{As}_{0,03} \mathrm{Sb}_{0,77}$. Par contre, ils sont très différents de ceux publiés par R. Chin et H. D. Law [13]. Ces derniers ont réalisé des diffusions de zinc en tube scellé sous vide à $520^{\circ} \mathrm{C}$, à partir d'une source constituée par $93 \mathrm{at}^{0 / 0}$ de gallium et $7 \mathrm{at}^{0 / 0}$ de zinc, et en présence de vapeurs d'antimoine. Les vitesses de diffusion sont beaucoup plus faibles, ce qui peut être attribué à la diminution de la pression partielle de zinc dans l'ampoule. Ce résultat a d'ailleurs été mis en évidence par A. I. Blashku et al. [11] qui ont étudié la diffusion de zinc dans GaSb fortement dopé. Les plus grandes profondeurs de pénétration ont été obtenues avec, comme source, du zinc pur, donc dans le cas où la pression de vapeur de zinc est la plus forte.

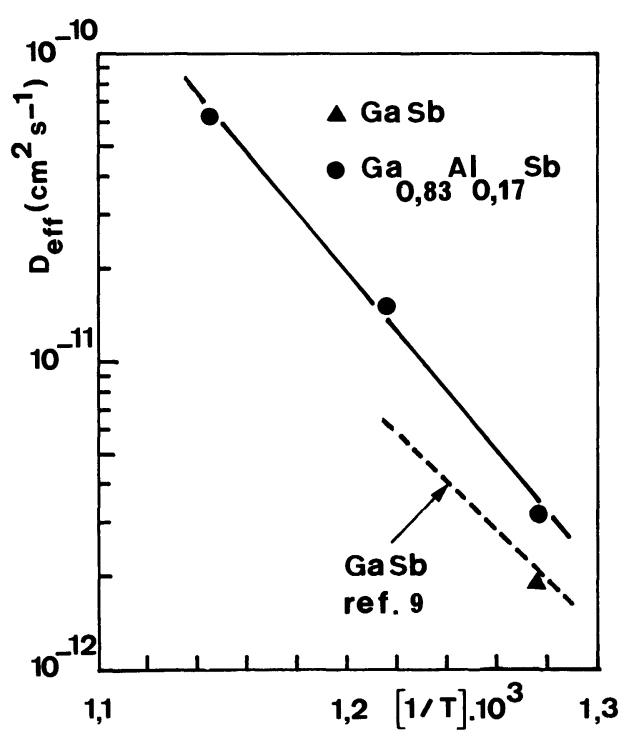

Fig. 8. - Variation du coefficient de diffusion effectif du zinc avec la température.

[Variation of the effective diffusion coefficient of zinc with the temperature.]
Dans les couches épitaxiées, le zinc diffuse moins vite dans $\mathrm{GaSb}$ que dans $\mathrm{GaAlSb}$. Ce comportement a été également observé pour le ternaire GaAlAs vis-à-vis du binaire GaAs [17].

Une interprétation possible de ce résultat est basée sur l'hypothèse de la réduction du nombre de lacunes dans les alliages ternaires - l'écart entre la température de fusion de l'alliage et la température de diffusion étant plus grand - ce qui réduit l'importance du mécanisme de diffusion substitutionnelle, au profit de la diffusion par mécanisme d'insertion [18, 19]. Ce dernier mécanisme étant plus rapide, il en résulte une augmentation de la vitesse de diffusion. Cette explication nous semble peu probable dans le cas des alliages GaAlSb. En effet, la réduction du nombre de lacunes par suite de la présence de l'aluminium devrait s'accompagner d'une diminution de la concentration en défauts résiduels. Cette diminution n'est pas observée avec le ternaire GaAlSb, cristallisé à la même température que GaSb. Les couches épitaxiées à $550^{\circ} \mathrm{C}$ possèdent un dopage résiduel $N_{\mathrm{A}} \sim 1,0 \times$ $10^{17} \mathrm{~cm}^{-3}$, indépendant de la composition de la couche.

Une autre explication peut être avancée. Elle tient compte des valeurs très élevées de la solubilité du zinc dans l'alliage. On sait que pour des concentrations d'impuretés élevées, la diffusion induit des défauts dans le cristal - défauts d'empilement, dislocations qui accélèrent le processus de diffusion [20]. Si la solubilité augmente avec la teneur en aluminium de l'alliage, le nombre de défauts induits va croître et, par conséquent, il en sera de même pour la vitesse de diffusion. La solubilité limite peut être évaluée dans notre cas en extrapolant la partie linéaire des profils de diffusion (Fig. 6) jusqu'à la surface. Les concentrations en zinc obtenues avec les alliages $\mathrm{Ga}_{0,83} \mathrm{Al}_{0,17} \mathrm{Sb}$ $\left(\simeq 10^{20} \mathrm{at} / \mathrm{cm}^{3}\right)$ sont effectivement supérieures à celles mesurées dans GaSb $\left(6-7 \times 10^{19} \mathrm{at} / \mathrm{cm}^{3}\right)$.

\section{Caractérisation photoélectrique des diodes diffusées.}

3.1 Réalisation Des PHOtodiodes. - Des diodes à structure MESA de diamètre $300 \mu \mathrm{m}$ ont été préparées par photolithographie sur les plaquettes diffusées. L'attaque chimique utilisée pour réaliser la gravure MESA est effectuée par une solution $2 \mathrm{HCl} / 1 \mathrm{CH}_{3} \mathrm{COOH} / 2 \mathrm{H}_{2} \mathrm{O}_{2}$ pendant $30 \mathrm{~s}$ ce qui assure une profondeur d'attaque de $10 \mu$.

La métallisation a été assurée par évaporation d'orzinc (avec le type $\mathrm{p}^{+}$) et d'or-germanium (avec le type $\mathrm{n}$ ), suivie d'un recuit à $350^{\circ} \mathrm{C}$ de $2 \mathrm{~min}$. La diode est ensuite montée sur un boîtier TO.5. Le contact sur la face $\mathrm{p}^{+}$est pris par collage à l'argent. La structure définitive est schématisée figure 9 .

3.2 CARACTÉRISTIQUeS ÉleCtriQues. - Les mesures de capacité montrent que les jonctions $\mathrm{p}^{+} \mathrm{n}$ obtenues sont abruptes, avec des dopages nets $N_{\mathrm{D}}-N_{\mathrm{A}}$ variant de 0,8 à $1,0 \times 10^{17} \mathrm{~cm}^{3}$. 


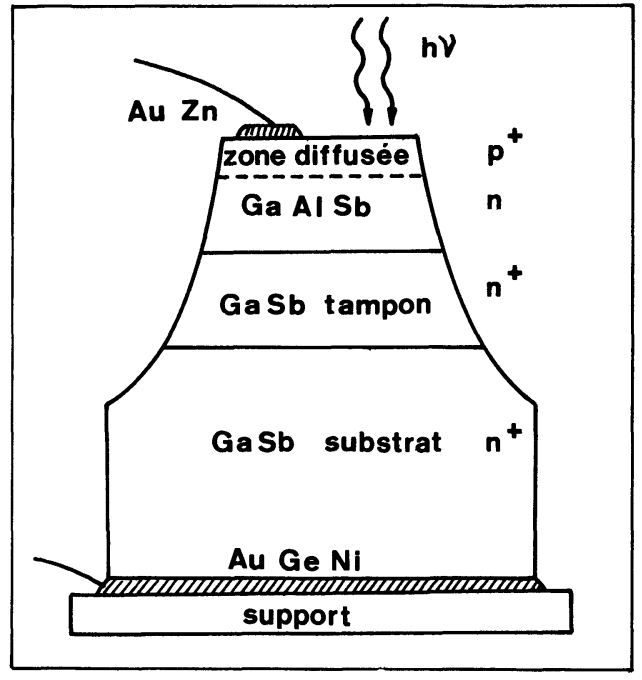

Fig. 9. - Schéma d'une diode MESA $\mathrm{p}^{+} \mathrm{n}$ à GaAlSb avec couche tampon.

[Scheme of the $\mathrm{p}^{+} \mathrm{n}$ MESA GaAlSb diode with a buffer layer.]

L'étude des caractéristiques courant-tension montre que le courant direct varie comme $\exp (q V / n k T)$ avèc $n \lesssim 2$. Les diodes présentent une résistance série de 5 à $10 \Omega$, une résistance shunt de l'ordre de $20 \mathrm{k} \Omega$.

En polarisation inverse, le claquage apparaît pour des tensions de 2 à $5 \mathrm{~V}$, valeurs compatibles avec le dopage $\mathrm{n}$ relativement élevé des couches épitaxiées $\left(10^{17} \mathrm{~cm}^{-3}\right)$. Le courant inverse est de $10 \mu \mathrm{A}$ à $-1 \mathrm{~V}$, ce qui correspond à une densité de courant de $10^{-2} \mathrm{~A} / \mathrm{cm}^{2}$. La valeur élevée de ce courant est attribuée à des courants de génération à partir de la surface, non passivée, de la diode MESA.

3.3 RéPONSE SPECTRALE. - Les mesures de réponse spectrale ont été effectuées en focalisant la lumière incidente, provenant d'une lampe à iode et filtrée par un monochromateur, de façon à obtenir un spot de 5 à $10 \mu \mathrm{m}$ de diamètre au niveau de la surface $\mathrm{p}^{+}$de la diode. La focalisation de la lumière incidente permet de s'affranchir des problèmes de bavures pendant la prise de contact par collage et de faire des comparaisons quantitatives entre différentes photodiodes.

On montre sur la figure 10 les réponses spectrales en courant de plusieurs types de diodes. Ces réponses sont identiques à polarisation nulle et à polarisation de $-1 \mathrm{~V}$. Sur cette figure, sont également reportés les rendements quantiques externes.

Le front de montée des courbes de réponse spectrale est abrupt. Ce résultat est dû à l'augmentation rapide du coefficient d'absorption avec la longueur d'onde, typique d'un matériau à gap direct. La légère diminution du signal observée immédiatement après le maximum est attribuée au phénomène de recombinaison en surface.

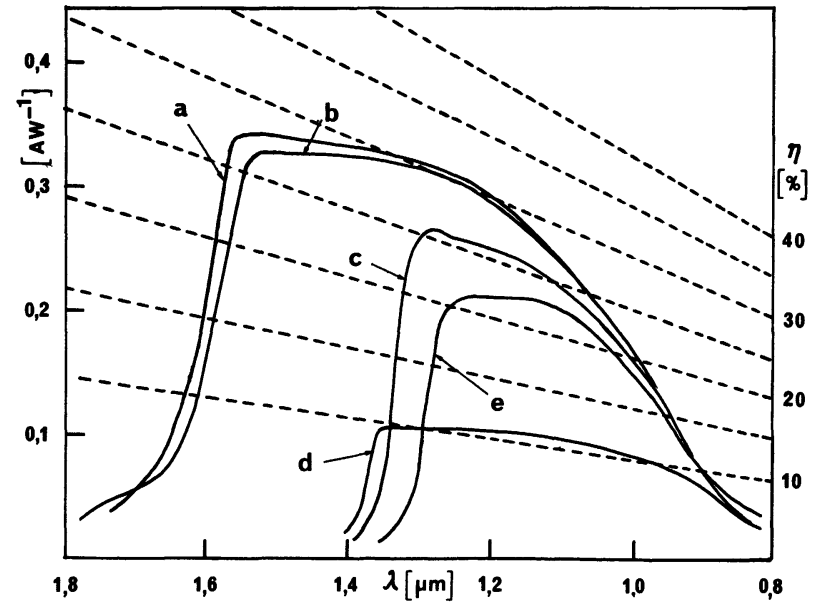

Fig. 10. - Réponse spectrale (A.W. ${ }^{-1}$ ) et rendement quantique de diverses diodes MESA diffusées à $\mathrm{Ga}_{1-x} \mathrm{Al}_{x} \mathrm{Sb}$.

a) $x=0,04$ durée de la diffusion $t=15 \mathrm{~min}$.
b) $x=0,07$
c) $x=0,15$
d) $x=0,15$
e) $x=0,17$
$\begin{array}{ll}- & - \\ - & - \\ - & -\end{array}$
$t=--$
$t=--$
$t=120 \mathrm{~min}$.
$t=30 \mathrm{~min}$.

[Spectral response (A.W. ${ }^{-1}$ ) and quantum efficiency of $\mathrm{Ga}_{1-x} \mathrm{Al}_{x} \mathrm{Sb}$ diffused MESA diodes :

a) $x=0.04$ diffusion time $=15 \mathrm{~min}$.

b) $x=0.07-\ldots$

c) $x=0.15 \quad-\quad--$
d) $x=0.15 \quad-120 \mathrm{~min}$

e) $x=0.17-\quad-\quad 30 \mathrm{~min}$.

La position du maximum de sensibilité est fonction de la composition de la couche, c'est-à-dire de son énergie de transition de bande interdite $E_{0}$ et se situe à une longueur d'onde $\lambda_{\max }$ légèrement inférieure à la longueur d'onde de coupure définie par $\lambda_{\mathrm{c}}(\mu \mathrm{m})=1,24 / E_{0}(\mathrm{eV})$. Ainsi, la réponse maximale est obtenue pour $\lambda=1,3 \mu \mathrm{m}$ et $1,55 \mu \mathrm{m}$ avec des échantillons de $\mathrm{Ga}_{1-x} \mathrm{Al}_{x} \mathrm{Sb}$ à $x=0,15$ et 0,04 donc à largeur de bande interdite $E_{0}=0,90 \mathrm{eV}$ et $0,77 \mathrm{eV}$ [21].

La figure 11 montre les valeurs du rendement quantique maximum pour des photodiodes à composition et dopages équivalents $(x=0,15$ à 0,17 et $N_{\mathrm{D}}-N_{\mathrm{A}} \sim 1,0 \times 10^{17} \mathrm{~cm}^{-3}$ ), mais à profondeurs de jonctions différentes. On constate une très nette augmentation des rendements quantiques pour des jonctions peu profondes. Malgré la dispersion des résultats, on peut voir que la loi de variation $\eta\left(x_{\mathbf{j}}\right)$ est exponentielle.

3.4 INTERPRÉTATION DES RÉSULTATS. - Le photocourant recueilli est la somme de trois composantes (Fig. 12) :- un photocourant $J_{1}$ dû à l'absorption de photons dans la couche $\mathrm{p}^{+}$de profondeur $x_{j}$. Ce photocourant est essentiellement un courant d'électrons dont l'expression en fonction de $x_{j}$ et du coeffi- 


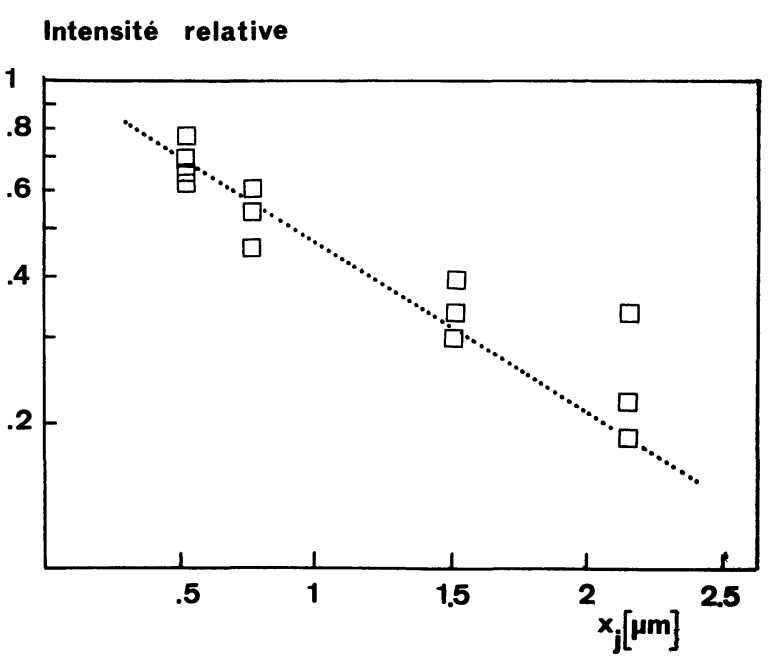

Fig. 11. - Relation entre la réponse maximale de diodes $\mathrm{p}^{+} \mathrm{n}$ à $\mathrm{Ga}_{0,83} \mathrm{Al}_{0,17} \mathrm{Sb}$ et la profondeur de jonction.

[Relation between the maximum spectral response of $\mathrm{p}^{+} \mathbf{n}$ $\mathrm{Ga}_{0.83} \mathrm{Al}_{0.17} \mathrm{Sb}$ diodes and the junction depth.]

cient d'absorption $\alpha$ est donnée par [22] :

$$
\begin{aligned}
& J_{1}=\frac{q \phi_{0}(1-R) \alpha L_{\mathrm{n}}}{\alpha^{2} L_{\mathrm{n}}^{2}-1}\left[-L_{\mathrm{n}} \exp \left(-\alpha x_{\mathrm{j}}\right)+\right. \\
& \frac{\left(\frac{S_{\mathrm{n}} L_{\mathrm{n}}}{D_{\mathrm{n}}}+\alpha L_{\mathrm{n}}\right)-\left(\frac{S_{\mathrm{n}} L_{\mathrm{n}}}{D_{\mathrm{n}}} \operatorname{ch} \frac{x_{\mathrm{j}}}{L_{\mathrm{n}}}+\operatorname{sh} \frac{x_{\mathrm{j}}}{L_{\mathrm{n}}}\right) \exp \left(-\alpha x_{\mathrm{j}}\right)}{\frac{S_{\mathrm{n}} L_{\mathrm{n}}}{D_{\mathrm{n}}} \operatorname{sh} \frac{x_{\mathrm{j}}}{L_{\mathrm{n}}}+\operatorname{ch} \frac{x_{\mathrm{j}}}{L_{\mathrm{p}}}}
\end{aligned}
$$

Dans cette expression, $\phi_{0}$ représente le flux de photons monochromatiques incidents, $R$ le coefficient de réflexion, $S_{\mathrm{n}}$ la vitesse de recombinaison des électrons à la surface, $L_{\mathrm{n}}$ la longueur de diffusion des électrons dans la zone diffusée, $D_{\mathrm{n}}$ le coefficient de diffusion de ces électrons.

- Un photocourant $J_{2}$ dû à l'absorption des photons dans la zone déplétée $W$. L'existence d'un champ électrique intense dans cette zone fait que toute absorption d'un rayonnement d'énergie supérieure au gap se traduit par un rendement quantique proche de 1 et un courant : $J_{2}=q \phi_{\mathrm{d}}[1-\exp (-\alpha W)]$ avec : $\omega_{\mathrm{d}}=$ flux de photons entrant dans la zone déplétée tel que

$\phi_{\mathrm{d}}=(1-R) \phi_{0} \exp \left(-\alpha x_{\mathrm{j}}\right)$

où $R$ est le pouvoir réflecteur de la surface $\mathrm{p}^{+}$

$\phi_{0}$ le flux de photons incidents.

- Un photocourant $J_{3}$ dû à la diffusion des trous photogénérés au-delà de la zone de charge d'espace et diffusant en direction de la jonction polarisée en inverse. On montre [23] par résolution de l'équation de diffusion, en se plaçant dans le cas semi-infini $\left(P_{\mathrm{n}}=P_{\mathrm{n} 0}\right.$ pour $\left.x=\infty\right)$, que :

$$
J_{3}=q \phi_{\mathrm{d}}\left(\frac{\alpha L_{\mathrm{P}}}{1+\alpha L_{\mathrm{P}}}\right) \exp \left[-\alpha\left(x_{\mathrm{j}}+w\right)\right]+q P_{\mathrm{n} 0} \frac{D_{\mathrm{P}}}{L_{\mathrm{P}}}
$$

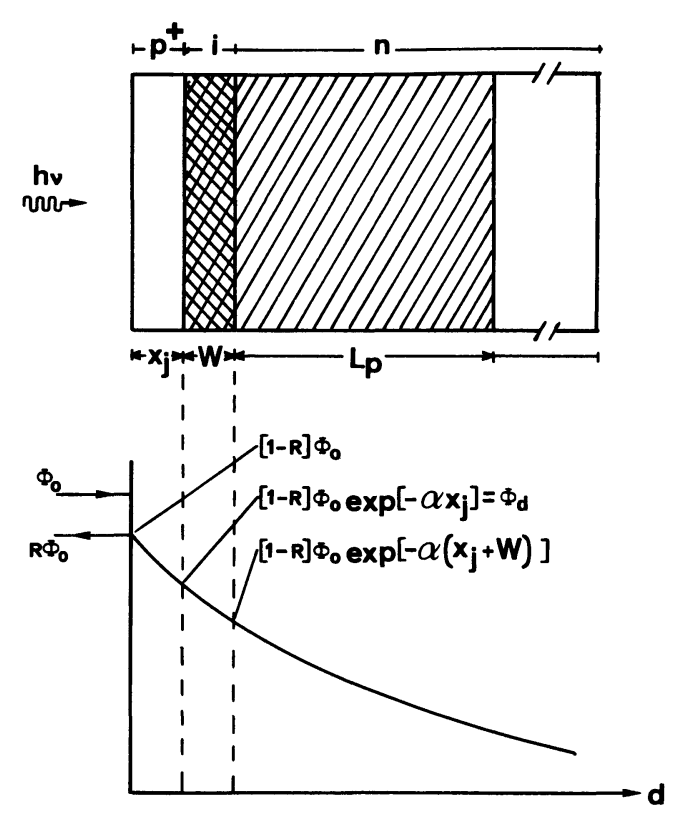

Fig. 12. - Absorption du rayonnement lumineux par une structure $\mathrm{p}^{+} \mathrm{n}$ polarisée en inverse. $\mathrm{p}^{+}$: zone diffusée; $\mathbf{i}:$ zone déplétée $; \mathbf{n}$ : couche épitaxiée non diffusée.

[Absorption of the light by a $\mathrm{p}^{+} \mathrm{n}$ structure in reverse bias. $\mathrm{p}^{+}$: diffused zone; $\mathrm{i}$ : depleted zone; $\mathrm{n}$ : non diffused epilayer.]

où $P_{\mathrm{n} 0}$ représente la concentration d'équilibre en trous dans la zone $n$.

$D_{\mathrm{P}}$ et $L_{\mathrm{P}}$ sont le coefficient de diffusion et la longueur de diffusion des trous.

On peut montrer que dans notre cas le second terme de cette expression est négligeable.

Les photocourants $J_{2}$ et $J_{3}$ sont proportionnels à $\exp \left(-\alpha x_{\mathrm{j}}\right)$, ce qui n'est pas le cas de $J_{1}$. La figure 11 montre que le rendement quantique externe $\eta=\left(J_{1}+J_{2}+J_{3}\right) / \mathrm{q} \phi_{0}$ varie expérimentalement d'une manière exponentielle avec $x_{\mathrm{j}}$. On peut en déduire que la zone frontale diffusée participe peu à la valeur maximum de la réponse spectrale. La pente de la droite fournit alors le coefficient d'absorption (pour $\lambda \sim 1,3 \mu \mathrm{m}$ ) :

$$
\alpha(1,3 \mu \mathrm{m})=1,1 \times 10^{4} \mathrm{~cm}^{-1} .
$$

\section{Conclusion.}

Le coefficient de diffusion du zinc dans $\mathrm{Ga}_{1-x} \mathrm{Al}_{x} \mathrm{Sb}$ entre $510^{\circ} \mathrm{C}$ et $600^{\circ} \mathrm{C}$ est donné par $D_{\text {eff }}=$ $D_{0} \exp \left(-E_{0} / k T\right)$, avec $x=0,17, E_{0}=1,92 \mathrm{eV}$ et $D_{0}=8 \mathrm{~cm}^{2} \mathrm{~s}^{-1}$. Les profondeurs de jonction obtenues sont supérieures ou égales à $0,4 \mu \mathrm{m}$. Les fronts de diffusion sont plats. La zone $\mathrm{p}^{+}$est très dopée $\left(>10^{20}\right.$ at. $\mathrm{cm}^{-3}$ ).

Le zinc diffuse plus rapidement dans l'alliage ternaire $\mathrm{GaAlSb}$ que dans $\mathrm{GaSb}$. Ce résultat est attribué au plus grand nombre de défauts induits créés en cours de diffusion par les valeurs très élevées de la solubilité limite du zinc dans GaAlSb. 
Des photodiodes à géométrie MESA ont été préparées par photogravure à partir de structures $\mathrm{p}^{+} \mathrm{n}$ diffusées.

L'étude de la réponse spectrale de ces diodes montre que le maximum de sensibilité est à $1,3 \mu \mathrm{m}$ pour les échantillons à $x=0,15(0,90 \mathrm{eV})$ et à $1,55 \mu \mathrm{m}$ pour les échantillons à $x=0,04(0,77 \mathrm{eV})$. Les rendements quantiques les plus élevés $(30 \%)$ sont obtenus avec les jonctions peu profondes $(0,4 \mu \mathrm{m})$.
Cette étude a permis d'évaluer le coefficient d'absorption de GaAlSb (à 1,3 $\mu \mathrm{m}): \alpha=1,1 \times 10^{4} \mathrm{~cm}^{-1}$

\section{Remerciements.}

Nous tenons à remercier Monsieur Claude Armand pour les analyses en sonde ionique qu'il a effectuées à l'INSA de Toulouse.

Ce travail a été soutenu par la convention DAII $\mathrm{n}^{\circ}$ 81-35039007909 $245 \mathrm{BCV}$.

Bibliographie

[1] Horiguchi, M., Electron. Lett. 12 (1976) 310.

[2] Payne, D. N. et Gambling, W. A., Electron. Lett. 11 (1975) 176.

[3] Law, H. D., Tomasetta, L. R., Nakano, K. et Harris, J. S., Appl. Phys. Lett. 33 (1978) 416.

[4] Law, H. D., Harris Jr., J. S., Wong, K. C. et TomaSETTA, L. R., 7e Int. Symp. on " GaAs and related Compounds ", St Louis, Missouri, 24-27 sept. 1978. Proc. in : The Institute of Physics Conf. Ser. $\mathrm{n}^{\circ} 45$, éd. C. Wolfe (1979) p. 420.

[5] Law, H. D., Chin, R., Nakano, K., Milano, R. A., I.E.E.E. J. Quant. Electron. QE 17 (1981) 275.

[6] Hildebrand, O., Kuebart, W., Benz, K. W. et PIlkuhn, M. H., I.E.E.E. J. Quant. Electron. QE 17 (1981) 284.

[7] Capasso, F., Hutchinson, A. L., Foy, P. W., Bethea, C. et Bonner, W. A., Appl. Phys. Lett. 39 (1981) 736.

[8] Chin, R. et Hill, C. M., Appl. Phys. Lett. 40 (1981) 332.

[9] Kagawa, T. et Motosugi, G., Japan J. Appl. Phys. 20 (1981) 597.

[10] Kyuregyan, A. S. et Stuchebnikov, V. M., Sov. Phys. Semicond. 4 (1971) 1365.

[11] BlashKu, A. I., Boltaks, B. I., Burdiyan, J. I., Dzha-
Farov, T. D. et Rzaev, M. A., Sov. Phys. Semicond. 6 (1972) 402.

[12] Da Cunha, S. F. et Bougnot, J., Phys. Status. Solidi (a) 22 (1974) 205

[13] Chin, R. et Law, H. D., J. Electrochem. Soc. 128 (1981) 227.

[14] Balyuba, V. I., Vilisov, A. A. et Germogenov, V. P., Izv. Vyssh. Uchebn. Zaved. Fiz. 22 (1979) 106.

[15] Ruprecht, H. et Le May, C. Z., J. Appl. Phys. 35 (1964) 1970.

[16] Casey, H. C. et Panish, M. B., Trans. Metal. Soc. AIME 242 (1968) 406.

[17] Lee, C. P., Maragalit, S. et Yariv, A., Solid State Electron. 21 (1978) 905.

[18] Longini, R. L., Solid. State Electron. 5 (1962) 127.

[19] Chang, L. L. et Pearson, G. L., J. Appl. Phys. 35 (1964) 1960.

[20] TuCK, B. et KadHIM, M., J. Mater. Sciences 7 (1972) 585.

[21] Alibert, C., Joullie, A. et Joullie, A. M., Phys. Rev. $B 27 \mathrm{n}^{\circ} 8$ (1983).

[22] LAUgier, A. et Roger, J. A., Les photopiles solaires, Technique et Documentation (1981) p. 96

[23] Sze, S. M., Physics of Semiconductor devices (WileyInterscience, Ed. John Wiley) 1969 p. 665. 